\title{
Low-Resolution Electromagnetic Tomography Neurofeedback
}

\author{
Marco Congedo, Joel F. Lubar, and David Joffe
}

\begin{abstract}
Through continuous feedback of the electroencephalogram (EEG) humans can learn how to shape their brain electrical activity in a desired direction. The technique is known as EEG biofeedback, or neurofeedback, and has been used since the late 1960s in research and clinical applications. A major limitation of neurofeedback relates to the limited information provided by a single or small number of electrodes placed on the scalp. We establish a method for extracting and feeding back intracranial current density and we carry out an experimental study to ascertain the ability of the participants to drive their own EEG power in a desired direction. To derive current density within the brain volume, we used the low-resolution electromagnetic tomography (LORETA).

Six undergraduate students (three males, three females) underwent tomographic neurofeedback (based on 19 electrodes placed according to the 10-20 system) to enhance the current density power ratio between the frequency bands $\beta(16-20 \mathrm{~Hz})$ and $\alpha$ $(8-10 \mathrm{~Hz})$. According to LORETA modeling, the region of interest corresponded to the Anterior Cingulate (cognitive division). The protocol was designed to improve the performance of the subjects on the dimension of sustained attention. Two hypotheses were tested: 1 ) that the $\beta / \alpha$ current density power ratio increased over sessions and 2) that by the end of the training subjects acquired the ability of increasing that ratio at will. Both hypotheses received substantial experimental support in this study.

This is the first application of an EEG inverse solution to neurofeedback. Possible applications of the technique include the treatment of epileptic foci, the rehabilitation of specific brain regions damaged as a consequence of traumatic brain injury and, in general, the training of any spatial specific cortical electrical activity. These findings may also have relevant consequences for the development of brain-computer interfaces.
\end{abstract}

Index Terms-Brain-computer interfaces (BCI), electroencephalogram (EEG) biofeedback, electromagnetic tomography, inverse solution, low-resolution electromagnetic tomography (LORETA), neurofeedback, $p$-value combination.

\section{INTRODUCTION}

$\mathbf{I}$ N THIS STUDY, we merge two widespread techniques used in electromagnetic neurosciences, electroencephalogram (EEG) biofeedback (neurofeedback) and low-resolution electromagnetic tomography (LORETA). The aim is to enhance

Manuscript received February 2, 2004; revised September 14, 2004. This work was supported in part by the International Society of Neuronal Regulation, in part by the Alumni Association of the University of Tennessee, and in part by Lexicor Medical Technology.

M. Congedo was with the Department of Psychology of the University of Tennessee, Knoxville, TN 37996-0900 USA. He is now with the Institute for Research in Informatics and Random Systems (IRISA), 35042 Rennes, France (e-mail: loretabiofeedback@yahoo.com).

J. F. Lubar is with the Department of Psychology, University of Tennessee, TN 37996-0900, USA.

D. Joffe is with Lexicor Medical Technology, Boulder, CO 80301 USA.

Digital Object Identifier 10.1109/TNSRE.2004.840492 the human ability to self-regulate brain electric activity. Neurofeedback is a technique used mainly in behavioral medicine as an adjunct to psychotherapy. An electronic device records EEG activity at a particular scalp location, extrapolates physiological measurements from the signal and converts them to visual and/or auditory representations dynamically covarying with the brain signals. The process is real-time, that is, the feedback modalities continuously represent brain activity with a minimum constant delay. Typically, over 20-40 sessions of 30 min each, spaced two to three days apart, the participant may acquire an enhanced awareness of the underlying brain processes and learn how to shape them in a desired direction. Research in this field commenced in the late 1960s (e.g., [1]-[3]) and focused mainly on the acquisition of control over the posterior dominant rhythm, 8-13 Hz, known as alpha. Clinical applications of neurofeedback have received much attention. Several successful protocols have been established for the treatment of attention deficit disorder [4]-[10], unipolar depression [11], and epilepsy [12]-[16]. Research is currently in progress relating to such disorders as traumatic brain injury [17], anxiety disorders [18], and chronic fatigue syndrome [19]. Similar learning processes related to the self-regulation of EEG have been recently reported in studies on brain-computer interfaces (BCIs, [20]).

Neurofeedback treatments do not always bring clinical improvements or rehabilitation. The most influential factor contributing to the success of neurofeedback training is probably the motivation of the participant, as argued in [6]. The neurofeedback process can be seen as a means of acquiring a certain degree of control over a physiological process. In fact, humans are not aware of their brain electrical activity directly, but rather through the associated conscious phenomena in the form of emotions, feelings, sensations, impressions, thoughts, and pain. Hereafter, by motivation, we refer to the volitional effort to achieve a result. In this sense, motivation belongs to consciousness and is synonymous with will. For instance [21], individuals are able to correctly estimate changes in their brain activity, corroborating the hypothesis that by means of feedback it is possible to acquire a certain awareness of physical brain processes. In other words, the control exercised upon a quantifiable phenomenon (electrophysiology) appears to be volitional, whereas in comparative research, e.g., [15], it has been shown to be susceptible to operant conditioning. In any case, how the brain achieves self-control by way of indirect feedback remains an open issue.

The most attractive qualities of neurofeedback as a clinical tool are that it is noninvasive and that it requires an active role on the part of the patient. A major limitation relates to the limited 
information provided by a single or small number of electrodes placed on the scalp. The response of any particular electrode is spatially unspecific. The EEG is produced by the measurable potential difference between the active electrodes and a reference location. The EEG signal mainly reflects the superposition of the electrical activity created by the ionic charge oscillation due to postsynaptic potentials of neocortical pyramidal cells (see [22] and [23]). Thus, a large population of neurons beneath an electrode is superimposed to create the measurable EEG but relatively little spatial information can be derived from a single channel of EEG. We argue that the learning process could be improved using more spatially specific information, which is achieved using an electromagnetic tomographic technique.

LORETA is a popular inverse solution technique. First reported in [24] and further expanded in [25]-[27], it has been recently reviewed in [28] and [29]. In its original formulation, or in a slight variant called the variable resolution electromagnetic tomography (VARETA), LORETA has been used extensively in applied electrophysiological research [30]-[33] and has been evaluated independently in several laboratories [34]-[37]. LORETA estimates the distribution of electrical neuronal activity in three-dimensional space, utilizing information acquired from a dense grid of electrodes placed over the entire scalp. While EEG is a measure of electric potential differences, LORETA estimates the current densities at depth which produce the measurable potential differences, or observed electric field on the scalp. In the LORETA-Key ${ }^{1}$ implementation of the LORETA method [27], the current density is estimated for 2394 voxels of dimension $7 \times 7 \times 7 \mathrm{~mm}$. The solution is restricted to gray matter (including part of the Hippocampus, Amygdala, and the entire Cingulate gyrus). LORETA-Key implements a three shell spherical model (skin, skull, and cortex) co-registered to the MRI atlas of Talairach and Tournoux [38]. Anatomical labeling of each grid point is then possible [39], [40]. The co-registration makes use of realistic EEG electrode coordinates reported in [41]. While traditional EEG information is limited to activity measured on the scalp, intracranial activity can be inferred through the use of inverse solution techniques. In [25], it has been shown that this inverse reconstruction is independent of the reference used in obtaining the EEG recordings as long as the reference is common to all electrodes. This is another distinctive advantage of linear inverse solutions over scalp EEG, which depends on the reference, because reference free methods yield more consistent results across laboratories.

Since neurofeedback is provided using electronic equipment, the whole field benefits from advances in technical instrumentation. Reference [42] explored functional magnetic resonance imaging (fMRI) neurofeedback. The aim of this study was to explore the use of LORETA current density data for neurofeedback. Possible applications of the techniques described herein include the treatment of epileptic foci, the rehabilitation of specific brain regions damaged as a consequence of traumatic brain injury, and the training of any specific cortical electrical activity in general. However, the technique is interesting beyond its clinic applications. Fields that may be using tomographic neurofeedback-like methods in the future include

${ }^{1}$ LORETA-Key is freeware software available from the Key-Institute for Brain-Mind Research, Zurich, Switzerland. virtual reality and BCIs. Recent advances in noncontact detection of human EEG using ultrahigh input impedance sensors [43] let us foresee in the near future a myriad of technological implementations. However, we need to establish the validity of the proposed method and this study constitutes the first step in this direction. Our contribution is twofold: we implemented the first LORETA tomographic neurofeedback system and we provided the first experimental evidence of learning based upon intracranial current density feedback. In the following sections, we describe both of these innovations.

\section{METHOD}

\section{A. Subjects}

Six undergraduate students (three males, three females, between 19 and 22 years old) volunteered to participate in the experiment in exchange for extra-credit and monetary compensation. They were selected according to exclusion and inclusion criteria and according to their interest in the research. Potential participants or their first-degree relatives were required to have no history of depression, anxiety, epilepsy, eating disorder, drug abuse, attention deficit, or any other psychiatric or neurological disorders that would confound their status as nonclinical healthy college students. The personal history and current habits of participants were assessed using a semistructured interview. In addition, we performed a comprehensive quantitative EEG evaluation comparing absolute and relative power in 13 standard bandpass regions at 19 electrodes (scalp voltage power) and all 2394 intracranial LORETA-Key voxels (LORETA current density power) to a normative database. This database was constructed using data acquired from 82 healthy undergraduate students from the University of Tennessee. We followed the nonparametric comparison method of [44]. Individuals displaying an abnormal number of significant (with $p<0.05$ ) deviations from the norms were excluded from the study. All participants were required to be alcohol and medication free for $24 \mathrm{~h}$ prior to sessions and were required to sign an informed consent form. All aspects of this study were approved by the University of Tennessee Human Subjects Review Board.

\section{B. Procedures}

For each subject, a measure of the distance between nasion and inion was used to determine the proper electrode cap [45] size for recording purposes. In preparation for neurofeedback sessions, both forehead and ears were cleaned using a mild abrasive gel to remove any oil from the skin. The cap was then fitted and each electrode site carefully injected with conductive gel. The scalp underlying each cap electrode location was mechanically abraded with the gel applicator so that impedances between each electrode site and each ear measured individually, as well as the impedance between both ears, was between 3 and $5 \mathrm{k} \Omega$. The EEG was then recorded at the standard 10-20 system 19 locations (FP1, FP2, F3, F4, Fz, F7, F8, C3, C4, Cz, T3, T4, T5, T6, P3, P4, Pz, O1, and O2) using the Neurosearch-24 EEG acquisition system. ${ }^{2}$ The EEG was sampled at $256 \mathrm{~Hz}$ and the

${ }^{2}$ Neurosearch-24 is produced and marketed by Lexicor Medical Technologies, Inc., Boulder, CO. 


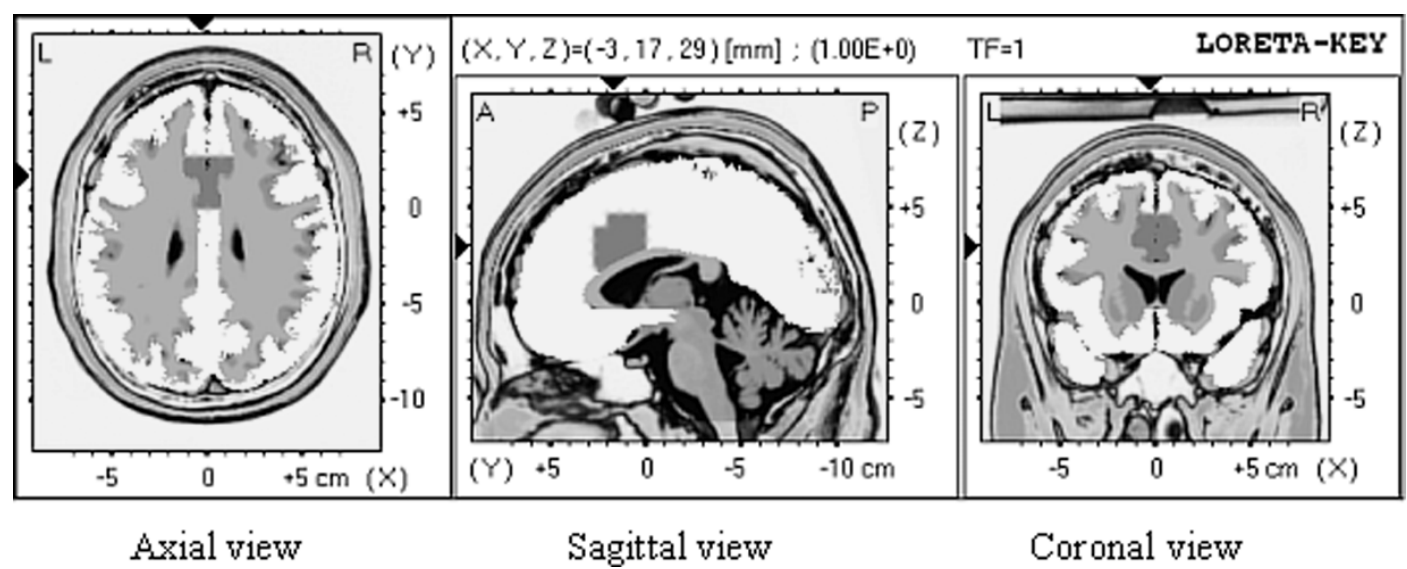

Fig. 1. Anterior Cingulate cognitive division (ACcd). The ROI used in LORETA modeling for training is shown with the LORETA-Key image viewer. ROI extension $=38$ voxels ( area $=13.034 \mathrm{~cm}^{2}$ ). Axial view: left of picture is left of the brain. The slice is seen from the top of the brain. Sagittal view: left of picture is front of the brain. The slice is seen from the left of the brain. Coronal view: left of picture is left of the brain. The slice is seen from the back of the brain.

low- and high-pass filters were set at $64-$ and $0.5-\mathrm{Hz}$ cutoffs, respectively.

\section{Protocol}

The Neurofeedack protocol was designed to enhance low beta $(16-20 \mathrm{~Hz})$ and suppress low alpha $(8-10 \mathrm{~Hz})$ current density amplitude (vector length) in a region corresponding approximately to the anterior cingulate cognitive division (ACcd). Hereafter, we will refer to the protocol using the symbol $\alpha-\mid \beta+$. The region of interest (ROI) is depicted in Fig. 1 using the LORETA-Key software. The region includes 38 voxels encompassing, according to the LORETA-Key head model, a total of $13.034 \mathrm{~cm}^{3}$. With other functional neuroimaging techniques such as positron emission tomography (PET) and fMRI, the ACcd has been consistently shown to be actively involved in attention processes [46], [47]. The choice of the bandpass regions was dictated by previous EEG studies. Converging evidence points to the role of frontal alpha and beta power in attention. A common pattern of abnormality in the electrophysiology of individuals affected by attention deficit disorder with (ADD) or without (ADHD) hyperactivity includes excessive low alpha power and weak low beta power [48]. Those individuals have a very short attention span and poor mental focusing capabilities, despite being of normal intelligence. On the other hand, alpha activity is known to decrease during cognitive functions [49] and to be inversely related to metabolism [50]. For over 30 years [6], the 16-20-Hz power enhancement protocol (in mid-frontal or mid-central locations $\mathrm{Fz}$ or $\mathrm{Cz}$ ) has been used to enhance attention in individuals suffering from ADD/ADHD. A merging of these lines of evidence resulted in our decision to suppress low alpha $(\alpha)$ and enhance low beta $(\beta)$ power with the aim of facilitating attention processes. Three subjects underwent six sessions consisting of six 3-min trials while the remaining three underwent 20 sessions of three 15-min trials. All parts of the experiment were conducted in a dimly illuminated and sound attenuated room at the Brain Research and Neurophysiology Laboratory, University of Tennessee. Two or three sessions were scheduled per week. For each individual, the time of the day for sessions was held constant.

\section{Physiological Signal}

In this section, we describe in detail the nature of the physiological signal (feature) extracted in real-time from the EEG. The feature we extracted has never been used before. We employed an $\alpha-\mid \beta+$ protocol, with alpha in the $8-10-\mathrm{Hz}$ range and beta in the $16-20-\mathrm{Hz}$ range. The peak-to-peak amplitude in both band-pass regions was continuously extracted in short time intervals $(250 \mathrm{~ms})$ through the use of digital filtering (see Section II-I.). A common choice (e.g., [6] and [9]) of the physiological signal for a $\alpha-\mid \beta+$ protocol is the $\alpha / \beta$ amplitude ratio. However, such a feature has several undesirable properties. Among others, its average level is subjective, as suggested in [51], and this precludes any direct intersubject comparisons. To overcome this limitation at each instant time $t$ we evaluated the (first-derivative) fractional changes $(\Delta)$ of $\alpha$ and $\beta$ expressed as

and

$$
\Delta \alpha_{t}=\frac{\left(\alpha_{t}-\alpha_{t-1}\right)}{\alpha_{t-1}}
$$

$$
\Delta \beta_{t}=\frac{\left(\beta_{t}-\beta_{t-1}\right)}{\beta_{t-1}}
$$

respectively. The ratio $\Delta \alpha / \Delta \beta$ is no longer a natural measure of the overall changes because the quantities $\Delta \alpha$ and $\Delta \beta$ are signed. Therefore, the $\Phi^{1}$ function is defined later to allow the extraction of a meaningful parameter relating to changes rather than raw measurements. Fig. 2 illustrates the changes in the two generic bandpass regions plotted in a Cartesian space where points have coordinates $[\Delta \alpha, \Delta \beta]$. The point in Fig. 2 corresponds to an approximately $25 \%$ decrease in alpha power and approximately $75 \%$ increase in beta power, hence it is found in the second quadrant. The polar coordinates $r=\sqrt{ }\left(\Delta \beta^{2}+\Delta \alpha^{2}\right)$ and $\theta=\operatorname{Tan}^{-1}(\Delta \beta / \Delta \alpha)$ represent the distance of the point from the origin, which is indicated by a segment labeled $r$, and the angle of the segment, indicated by $\theta$.

For a $\alpha-\mid \beta+$ protocol we wish to use a function that increases monotonically as $\Delta \beta$ increases and decreases monotonically as $\Delta \alpha$ increases. Without loss of generality, a protocol involving two bandpass regions can be arranged changing the angular coordinate $\gamma$ of $\theta$ and evaluating $\operatorname{Sin}(\theta-\gamma)$. In this manner, we can 


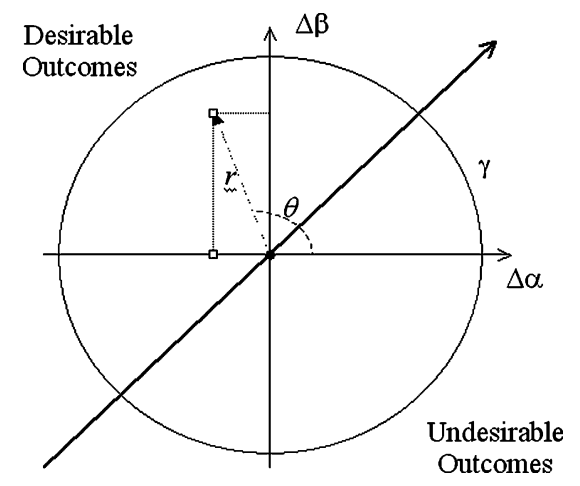

Fig. 2. Plot of fractional changes $\Delta \alpha$ and $\Delta \beta$ in a two-dimensional space. Any measurement of fractional changes $\Delta \alpha$ and $\Delta \beta$ can be represented as a point with polar coordinates $r$ and $\theta$, where $r$ represents the strength of the change and $\theta$ represents the direction of the change. The angular coordinate $\gamma$ depends on the protocol employed. Mapping $\Delta \alpha$ and $\Delta \beta$ to the abscissa and ordinates respectively, for a $\alpha-\mid \beta+$ protocol, $\gamma$ equals $45^{\circ}$. The new abscissa is represented as a thick line. Due to the rotation, $\operatorname{Sin}(\theta-\gamma)$ reaches its maximum when $\Delta \beta$ increases as $\Delta \alpha$ decreases (most desirable event), and its minimum when the opposite is true (most undesirable event).

conveniently map any direction of change for any ratio protocol. In the particular case of this $\alpha-\mid \beta+$ study, since $\Delta \alpha$ and $\Delta \beta$ have been mapped to the abscissa and ordinates, respectively, $\gamma$ has to be equal to $45^{\circ}$. This is seen graphically in Fig. 2, where the thick line is the rotated abscissa. Thanks to the rotation, the quantity $\operatorname{Sin}(\theta-\gamma)$ reaches its maximum (1.0) when beta decreases as alpha decreases, and its minimum $(-1.0)$ for the opposite outcome. Those are defined as the most desirable and the most undesirable outcomes respectively, according to the protocol we chose. In order to account for the strength of the outcome and to obtain a function with bounded dynamical range, we also considered the negative exponential function $\left(1-e^{-r}\right)$, which assumes a zero value when $r$ is zero and reaches 1.0 as $r$ approaches infinity. The $\Phi^{1}$ function is defined by multiplying the sine of $(\theta-\gamma)$ by this exponential function to obtain the function

$$
\Phi^{1}=\left(1-e^{-r}\right) \operatorname{Sin}(\theta-\gamma)
$$

bounded between -1.0 and 1.0. With two parameters $r$ and $\theta$, the function unfolds in three dimensions. Fig. 3 illustrates the mesh plot of the function $\Phi^{1}$ expressed with the original $\Delta \alpha$ and $\Delta \beta$ coordinates. Both axes are restricted to \pm 7.0 , the equivalent of a $\pm 700 \%$ change in alpha and beta. In Fig. 3 the $x$ axis is $\Delta \alpha$, while the $y$ axis is $\Delta \beta$. The $z$ axis is the color-coded value of $\Phi^{1}$, which is clearly shown to be a function of the axes rotated by $\gamma\left(45^{\circ}\right)$. In the plot, white corresponds to the maximum of $\Phi^{1}$, whereas black corresponds to its minimum. $\Phi^{1}$ reaches its maximum faster for $\theta=135^{\circ}$, and the minimum for $\theta=315^{\circ}$. For values of $\operatorname{Sin}(\theta-\gamma)$ close to zero $\left(\theta \approx 45^{\circ}\right.$ and $\left.\theta \approx 225^{\circ}\right)$, when desirable changes in beta are compromised by undesirable changes in alpha and vice versa, the function reaches its maximum extremely slowly, meaning that a very high value of $r$ is required for the attainment of a high value of $\Phi^{1}$.

If the brain was a homeostatic system with fractional changes comparable across individuals, then the expected value of $\Phi^{1}$ should be zero. In other words, since changes in the two bandpass regions of interest cannot continue indefinitely in the positive (increase in power) or negative (decrease in power) direction, and assuming that changes in one direction will be simi-

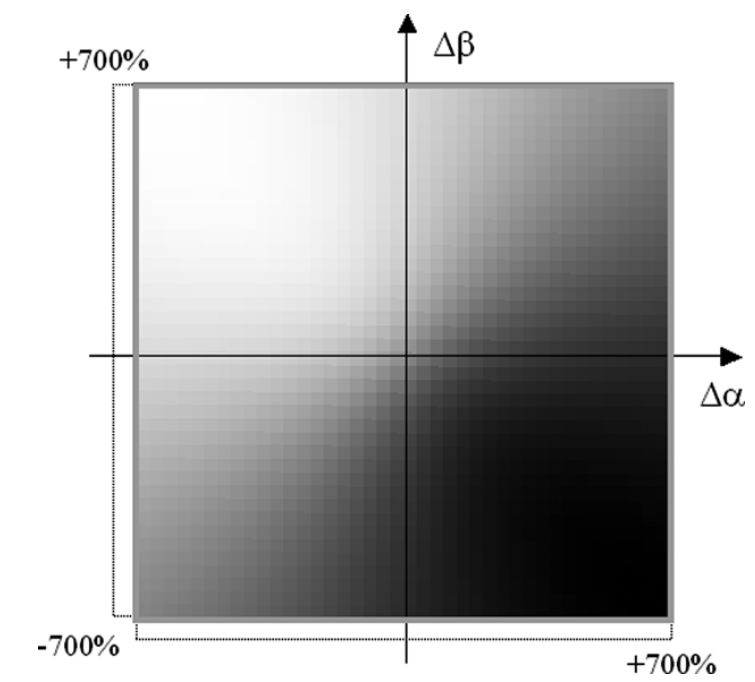

Fig. 3. Contour plot of the $\boldsymbol{\Phi}^{1}$ function. The $x$ axis is $\Delta \alpha$ and the $y$ axis is $\Delta \beta$. Both axes in the plot are limited by a $\pm 700 \%$ change. The $z$ axis is the color-coded value of $\Phi^{1}$, which is a function of the axes rotated by $\gamma\left(45^{\circ}\right)$. White $=\operatorname{Max}\left(\Phi^{1}\right)=1.0$. Black $=\operatorname{Min}\left(\Phi^{1}\right)=-1.0$. Changes $\Delta \alpha$ and $\Delta \beta$ are given by formula (1) and (2). The $\Phi^{1}$ is given by formula (3). See text for details.

larly compensated for by changes in the opposite direction so as to maintain a sort of symmetric homeostasis, then over a sufficiently long period of time the realization of $\Phi^{1}$ will lead to a sample mean of zero. Our empirical observation is that $\Phi^{1}$ for alpha and beta oscillates with sample means close to but not equal to zero. This is due to the fact that the empirical distribution of the $\Phi^{1}$ function is not exactly symmetric and suggests that positive and negative changes are subject to different physiological dynamics. In other words, while increases and decreases in amplitude are balanced in the long run, they do not follow the same distribution with respect to each other. However, the distribution of $\Phi^{1}$ is very similar across individuals, but the distribution of the $\beta / \alpha$ power ratio is not, hence by using the $\Phi^{1}$ function we achieve data normalization across individuals. This property of $\Phi^{1}$ is further discussed in the following section.

\section{E. Feedback Signal}

The neurofeedback literature makes a distinction between a self-regulation and an operant conditioning paradigm. Self-regulation stresses the importance of motivation in achieving a result and seems to be facilitated by the use of a continuous feedback signal [52]. Traditional operant conditioning, in contrast, requires the use of a discrete reinforcement [14] to reward changes in the predefined direction. We shall refer to the continuous feedback signal as CFS and to the discrete feedback signal as DFS. In our experiments, we used both auditory and visual CFS and DFS. Visual CFS was provided in the form of a moving scatter plot where each value of $\Phi^{1}$ was represented by a square. Fig. 4 shows a snapshot of the subjects' screen during LORETA neurofeedback. The horizontal line in the middle of the moving scatter plot corresponds to a zero value. Dots above the line indicate positive values (desirable changes), while dots below the line indicate negative values (undesirable changes). The auditory CFS implemented was a short $(100 \mathrm{~ms})$ tone whose pitch increased proportionally to $\Phi^{1}$. The range of the $\Phi^{1}$ function was divided into 21 equal intervals. Each interval, 
from the lower to the upper, corresponded to an increasing pitch ranging over the 21 notes comprising three octaves of the C-major scale. In all our experiments, the $\Phi^{1}$ function was updated every $250 \mathrm{~ms}$. Feedback provided at such a short interval can be considered continuous.

Visual DFS was provided in the form of a large flashing light on the screen (Fig. 4). The color of the flashing light, as with all other feedback settings, was chosen by the participants during a pilot session so as to be subjectively pleasant. They were also able to choose between visual and/or auditory feedback modalities with one single constraint: at least one continuous and one discrete modality had to be selected. The auditory DFS consisted of a short melody formed by a fast sequence of three tones, the total duration of which was less than $600 \mathrm{~ms}$. The auditory DFS was easily discernible from the auditory CFS due to its distinct duration and fixed sequence of tones. All DFS (light flashes or sequence of tones) were delivered as soon as the reinforcement conditions were matched. In all our experimental studies, the conditions to deliver a reinforcement at any instant time $t$ were the same, and exactly: $\Phi_{t}^{1}>0.1$ and $\Phi_{t-1}^{1}>0.1$. The $\Phi^{1}$ function constantly oscillated between positive and negative values. The conditions we set for reinforcement required that the individual maintained a desirable change for at least 500 ms. The objective was to train individuals to sustain changes in the desired direction. The 0.1 threshold was introduced in order to suppress noise. We carried out a total of six experiments using the above protocol. For all six individuals, the percent time spent in the reinforcement state, as seen in preneurofeedback baselines, was around $10 \%$. We can finally point to a critical advantage of the $\Phi^{1}$ function: the same threshold and conditions for reinforcement could be used for all individuals. As implied in the previous section, the use of traditional features such as raw amplitude and amplitude ratio does not allow for a common threshold to be applied to several individuals attaining similar percent times in the reinforcement state.

\section{F. Inhibition Filters}

When working with the EEG in general, and especially in real-time applications, it is extremely important to address the problem of extra-cranial sources of artifacts [53]. EEG artifacts can introduce a large amount of noise into the signal and, thus, can invalidate the generation of inverse solutions. Among others, two families of artifacts represent a serious problem for real-time applications; electromyography (EMG) refers to electrical activity generated by muscle contraction. Jaw, neck, shoulders, forehead, and other facial muscle activity are easily recorded from electrodes on the scalp resulting in low auto-correlation high-frequency noise that masks the signal. Eye movements, blinks, etc. [electrooculogram (EOG)], produce low-frequency high-amplitude potentials.

In order to limit the influence of these artifacts, we implemented two inhibition filters that constantly monitored the EMG and EOG activity during all neurofeedback sessions. Anytime the EMG or EOG signal exceeded its threshold, the corresponding inhibit filters turned on and interrupted the feedback loop. The loop resumed one second subsequent to the inactivation of the inhibition filters. The status of each filter was communicated to the subject by means of secondary

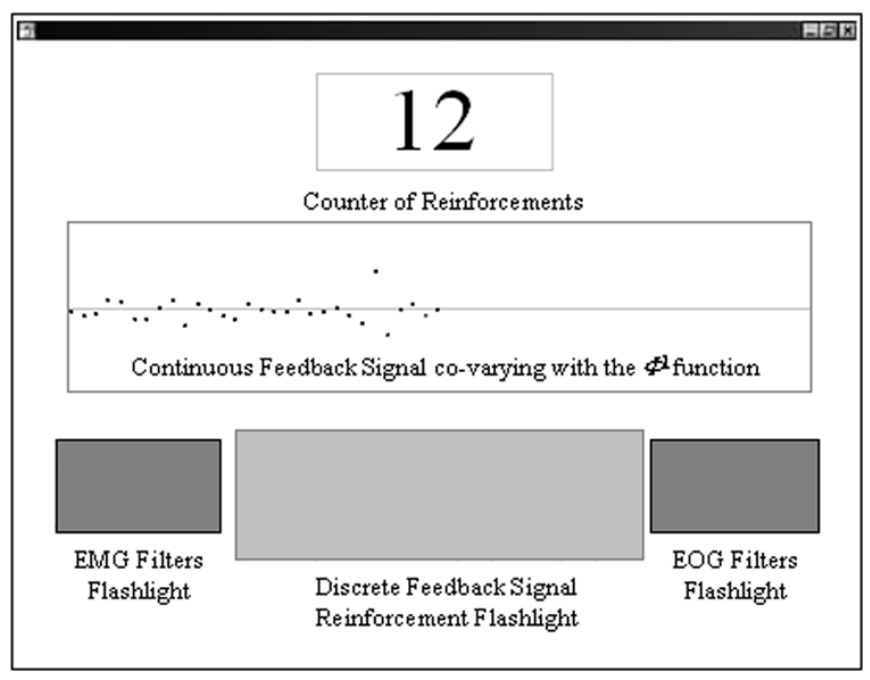

Fig. 4. Snapshot of the LORETA neurofeedback program interface. This is an example of the computer screen as seen by the participants during neurofeedback. The interface is controlled by the server program (see Section II-I.). The flashlights of the EMG inhibition filter, EOG inhibition filter and the DFS reinforcement flashlight are shown in a putative "on" position. With these color settings, they would turn white if in "off" position.

flashing lights on the screen (Fig. 4). We observed that, thanks to the flashing lights, by the end of the first session all subjects acquired an awareness of the causes of the filters activation. Thereafter, subjects were able to reduce the production of facial EMG and EOG activity to a minimum.

Thresholds for EMG and EOG were established and set in a pilot session for each individual separately. EMG activity was detected by computing the average LORETA current density in the ROI in the 35-55 Hz EEG filtered band (EMG channel). EOG activity was detected by measuring the maximum absolute voltage across frontal electrodes FP1, FP2, F7, F8, F4, and F3 (EOG channel). Fig. 5 shows time series of the second 15-min trial for the second session of subject 4. Data points are measurements continuously extracted every $250 \mathrm{~ms}$. (see Section II-I for details on data extraction) while both inhibition filters were off. Fig. 5(a) plots the $\beta / \alpha$ current density power ratio in the ROI (target channel). Fig. 5(b) and (c) plot the corresponding EMG and EOG channel, respectively. Fig. 5(d)-(f) are all possible pair-wise $( \pm 10 \mathrm{~s})$ cross-correlation plots of the target, EMG and EOG channels. In this example of training data, we can see that, after suppressing suprathreshold data containing excessive artifacts, the time course of the target feature $(\beta / \alpha$ ratio) was poorly correlated to EMG and EOG artifacts. However, since for no time lag and short time lags some correlation exists, we treated the EMG and EOG measurement as covariates (see Section II-H).

\section{G. Statistical Data Analysis}

Neurofeedback experiments usually involve a collection of single-case experiments as the learning process is unique to each individual. There is no reason to expect the learning curve of an individual to be congruent with the learning curve of another. This is one of those experimental situations in which average group analysis is of questionable value. Instead of averaging the data from all individuals we ran single-case statistical tests and then combined the $p$-values obtained across individuals. A combination of $n p$-values is a $p$-value itself. It is the probability of 
A: Target Channel

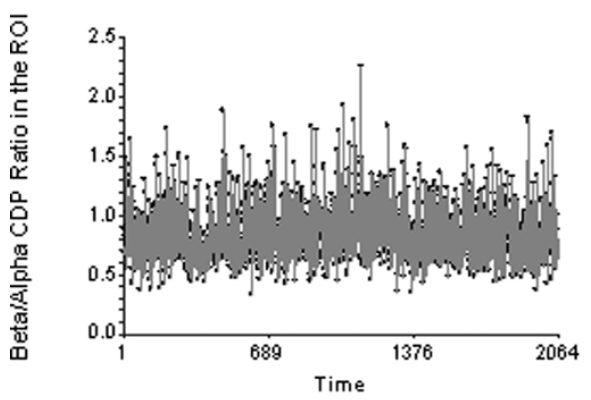

D: Target Channel vs. EMG Channel

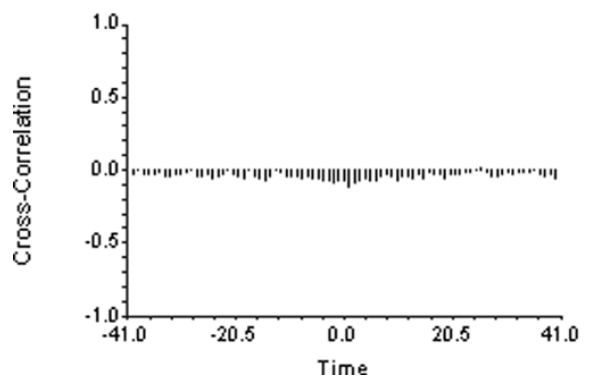

B: EMG Channel

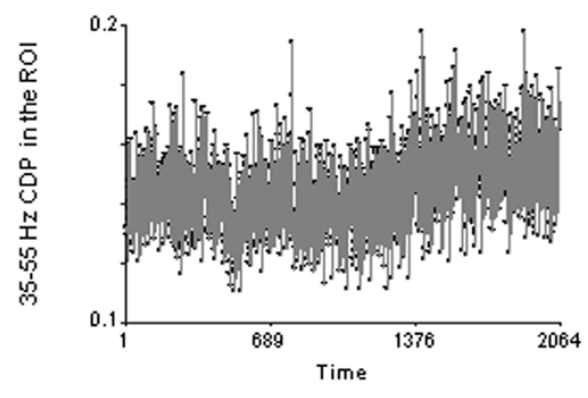

E: Target Channel vs. EOG Channel

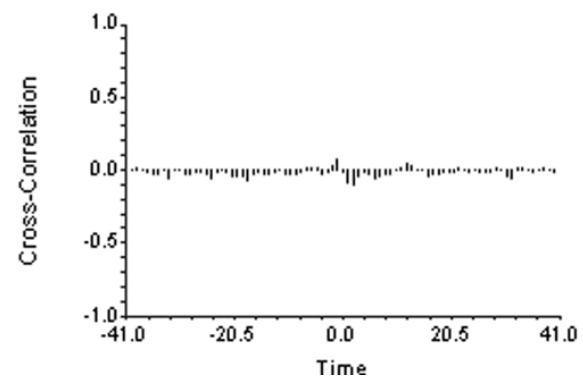

C: EOG Channel

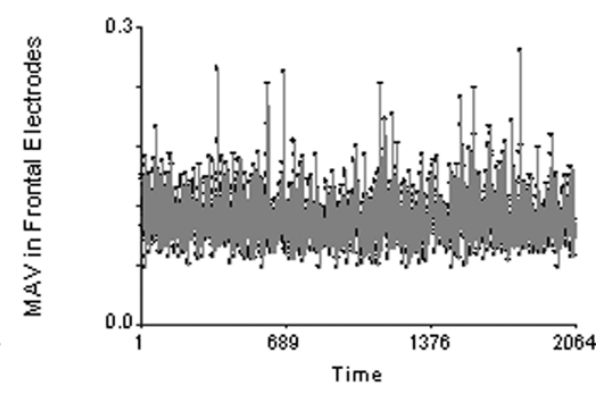

F: EMG Channel vs. EOG Channel

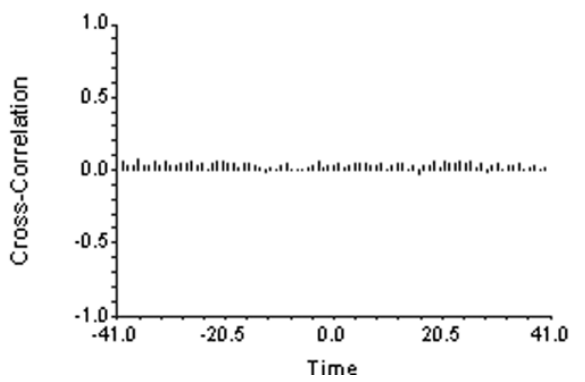

Fig. 5. Example time series of the artifact-free training session data. Time series of the $\beta / \alpha$ current density power (CDP) ratio in the ROI (A: target channel), 35-55 Hz current density power in the ROI (B: EMG channel) and maximum absolute voltage (MAV) at frontal electrodes FP1, FP2, F7, F8, F4, and F3 (C: EOG channel). Data refer to the second round of session 2 for subject 4 . Measurements were extracted every $250 \mathrm{~ms}$ and the process was suspended anytime either the EMG or the EOG inhibition filter turned on. D, E, and F are cross-correlation plots obtained lagging the time series from around $-10 \mathrm{~s}$ to around $+10 \mathrm{~s}$. The trial lasted approximately $15 \mathrm{~min}$, of which, 8.6 (2064 samples) were used for data analysis. These were the periods when no inhibition filters detected artifacts.

obtaining $n p$-values as small as those observed, given that the complete null hypothesis was true. This concept is similar to that of multivariate testing, where a unique $p$-value is derived for all variables. The most important advantage of this approach is that it does not require the experimental conditions to be identical for all subjects, nor does it assume that the learning process is the same in different individuals. Finally, the sample size is not a concern in $p$-value combination. The only assumption of (parametric) $p$-value combination is that the $p$-values all be pair-wise independent. Since individuals are truly independent statistical entities, deriving $p$-values for each subject separately is always a valid approach.

There exist several parametric methods that can be used to combine $p$-values. In our experimental studies, we systematically used two of them, namely the multiplicative combining function due to Fisher [55] and the additive combining function of Edgington [54]. The multiplicative combining function $P^{\times}$ is given by

$$
P^{\times}=\chi_{2 \mathrm{n}}^{-1}(F)
$$

where $\chi_{2 \mathrm{n}}^{-1}$ is the inverse c.d.f. of a central Chi-Square distribution with $2 n$ degrees of freedom, and $F=-2 \sum_{i=1}^{n} \ln \left(p_{i}\right)$.

The additive combining function $P^{+}$is directly calculated as

$$
\begin{aligned}
P^{+}=\frac{1}{n !}\left[C_{(n, 0)}(S-0)^{n}-C_{n, 1}(S-1)^{n}\right. & \\
& \left.+C(n, 2)(S-2)^{n}, \ldots,\right]
\end{aligned}
$$

where $C(n, k)$ is the number of combinations drawing $k$ items out of $n, S=\sum_{i=1}^{n} p_{i}$, the minus and plus signs between terms alternate, and additional terms are used as long as the number subtracted from $S$ is smaller than $S$. In all of the expressions, $p_{i}$ indicates the individual $p$-value.

We chose to use both of these functions because they display distinct sensitivities for extreme distributions of $p$-values. In particular, the multiplicative combining function is more sensitive to distributions where there is one or more extremely small $p$-value. The additive combining function is more sensitive when the $p$-values are all similar to each other. For combined $p$-values we set the type I error to 0.05 , but to claim significance we required that both combining functions produce a $p$-value less then or equal to 0.05 .

\section{H. Experimental Hypotheses}

To test hypotheses regarding the modification of brain electrical activity we focused on two distinct types of learning associated with neurofeedback. The first, here called exercise learning (EL), describes a monotonic trend of the target activity over sessions. It shows that the brain activity of the participants, as recorded during the sessions, tends to increase (or decrease) as a function of the number of sessions. The second, called volitional control (VC), describes the acquired ability of the participant to shape his or her target brain activity in the desired direction at will. EL could be achieved through either motivation (self-regulation) or mere operant conditioning. On the other hand, the test on VC that we here introduce, rules out any influence of unconscious processes. Regarding the EL hypothesis, we analyzed five dependent variables. For each participant and for each neurofeedback session, we extracted

1) the median of the average $\alpha$ amplitude in the ROI;

2) the median of the average $\beta$ amplitude in the ROI;

3) the $\beta / \alpha$ ratio $(2 / 1)$; 
4) the median of the $\Phi^{1}$ function;

5) the percent time spent in reinforcement state.

Within each session, the median was computed as the mean of the median of the signal continuously extracted during each trial. We also extracted the median EMG and EOG in the same way. Those two variables were used as covariates. For the VC hypothesis, all participants underwent eight 3-min trials at the end of the experiment. In four of them, labeled as "A," the participants were instructed to obtain as much reinforcement as possible. In the remaining four trials ("B"), the participants were instructed to obtain as little reinforcement as possible. The participants underwent the trials according to the same protocol employed during the neurofeedback sessions. The order of A and $B$ trials was randomized by shuffling eight cards, of which four reported the letter $\mathrm{A}$, and four reported the letter $\mathrm{B}$. The random order was established once and applied to all subjects. It was: A, B, A, B, B, B, A, A. The dependent variables analyzed were the same as those analyzed for the EL hypothesis. For both the EL and VC all dependent variables were analyzed by means of an exact randomization ordinary least square (OLS) linear regression model [56] as computed using the BLOSSOM statistical package [57]. In both cases, EMG and EOG were treated as covariates in order to control for their confounding influence. The test procedure implemented in the program is the randomization-permutation equivalent of an analysis of covariance (ANCOVA) and is explained in [58] and [59]. We stress the importance of considering EEG artifacts as confounding variables in this kind of experiment. Since artifacts may result in the modification of the target feature, there exists a risk that learning processes will be reported due simply to increased/decreased artifactual activity. Note that in addition to feeding back the $\Phi^{1}$ function, we also analyzed the $\beta / \alpha$ amplitude ratio, and the $\beta$ and $\alpha$ amplitude as well.

\section{Equipment}

Tomographic neurofeedback was implemented using two linked computers. The first, hereafter referred to as the server, was interfaced to the EEG preamplifiers in order to acquire 23 channels of raw physiological data (19 EEG and 4 auxiliary) at a sample rate of $512 \mathrm{~Hz}$. After suitable amplification, high-pass filtering to reject dc offset, and notch filtering (to eliminate $60-\mathrm{Hz}$ power line interference), each channel was then subjected to analog eighth-order lowpass maximally flat Butterworth filters in the bandpass $(70-\mathrm{Hz} 3-\mathrm{db}$ cutoff) for anti-aliasing purposes prior to analog/digital conversion. Each of the 23 channels of data was then lowpass digital filtered and resampled in order to derive 23 channels at a sample rate of $256 \mathrm{~Hz}$ (80-Hz 3-db cutoff). This produced 23 data streams with a closely matched interchannel phase response and overall filter characteristics. The server program allowed for the specification of three maximally flat (in the bandpass region) Butterworth bandpass filters. Nineteen of the 23 resampled channels of incoming data, those corresponding to the active EEG electrodes, were then subjected to real-time digital bandpass filtering, which resulted in three parallel streams of 19 channel data. In these experiments the filters were set to alpha, beta, and EMG bandwidths, as defined previously in the paper. The LORETA transformation (inversion) matrix values were extracted from the LORETA-Key package, and loaded into the server software prior to data acquisition. During real-time data acquisition, the portions of the LORETA-Key transformation matrix corresponding to the predefined ROI were then applied to the real-time 19 channel EEG data stream output of the three bandpass filters described earlier, on a sample by sample basis, thus producing an alpha, beta, and EMG current density amplitude value for each voxel in the ROI. Current density amplitude is defined as the square root of the sum of the square of each dipole basis moment $x, y$, and $z$ (vector length). The signal value at each voxel in each of the three digitally filtered ROI bands was then subjected to smoothing using a simple moving average circular buffer technique, and a running average value was computed for each voxel in the ROI, for each band. Finally, the average power for each band in the ROI was calculated, scaled, and then transmitted over a serial connection to the client every $250 \mathrm{~ms}$.

The job of the second computer, the client, was to compute the target feature (Section II-D), deliver the appropriate visual and/or auditory feedback to the participant (Section II-E), and manage the inhibition filters (Section II-F). The client computer also took responsibility for storing data for subsequent analysis. The server-client system involved two independent computer monitors, one for the experimenter and one for the participant. The server monitor was continuously observed by the experimenter and displayed the raw EEG in real-time. In this manner, the experimenter could verify the behavior of the inhibition filters, and insure that other sources of artifact did not appear such as those that might result, for example, from a broken electrode/skin contact. The client software reported only artifact-free data, that is, while the EMG or EOG filter was on, the program suspended data storage.

\section{RESULTS}

We tested the hypothesis that the participants attained VC (volitional control) and EL (exercise learning). A priori hypotheses for the direction of results were available for all tests, based on the protocol employed. Therefore, all tests were one-tailed and, for all results, a combined $p$-value smaller than 0.5 indicates evidence supporting the alternative hypothesis, whereas a combined $p$-value larger than 0.5 argues against the alternative hypothesis. Individual and combined results for VC are presented in Table I. For all dependent variables, observed changes between trials A and B were in the desired direction according to both combining functions. At the individual level, depending on the dependent measure, results were in the desired direction for four to six subjects. The major dependent variable of this study, the $\beta / \alpha$ amplitude ratio, was significantly larger during trials $\mathrm{A}$ as compared to trials $\mathrm{B}$, that is, overall, participants were successful in generating an increase of the $\beta / \alpha$ ratio at will $\left(P^{+}=0.012 ; P^{\times}=0.002\right)$. At the individual level, the $p$-values were significant for three out of six subjects. Results pertaining to the $\beta$ and $\alpha$ amplitude alone suggest that the change was predominantly driven by an increase in the $\beta$ amplitude, although decrease in $\alpha$ was, overall, also marginally significant. Neither the percent time spent in reinforcement state nor the $\Phi^{1}$ function itself were significantly affected by 
TABLE I

RESULTS OF THE VC HYPOTHESIS

\begin{tabular}{r|c|c|c|c|c|c|c|c}
\hline VC; $\mathrm{n}=6$ & $\mathrm{~S} 1$ & $\mathrm{~S} 2$ & $\mathrm{~S} 3$ & $\mathrm{~S} 4$ & $\mathrm{~S} 5$ & $\mathrm{~S} 6$ & $\mathbf{P}^{+}$ & $\mathbf{P}^{\mathbf{x}}$ \\
\hline$\alpha$ & 0.2877 & 0.2381 & 0.0142 & 0.5848 & 0.0647 & 0.9155 & $\mathbf{0 . 1 0 6}$ & $\mathbf{0 . 0 5 7}$ \\
\hline$\beta^{*}$ & 0.1230 & 0.2080 & 0.0003 & 0.3811 & 0.2866 & 0.4947 & $\mathbf{0 . 0 0 3}$ & $\mathbf{0 . 0 1 5}$ \\
\hline$\beta / \alpha^{*}$ & 0.2940 & 0.0160 & 0.0051 & 0.5819 & 0.0222 & 0.6415 & $\mathbf{0 . 0 1 2}$ & $\mathbf{0 . 0 0 2}$ \\
\hline$\Phi^{l}$ & 0.0763 & 0.2336 & 0.4083 & 0.7800 & 0.3403 & 0.4510 & $\mathbf{0 . 1 6 2}$ & $\mathbf{0 . 2 9 5}$ \\
\hline$\% \mathrm{tRS}$ & 0.0545 & 0.3090 & 0.3158 & 0.2901 & 0.5801 & 0.2305 & $\mathbf{0 . 0 4 2}$ & $\mathbf{0 . 1 5 1}$ \\
\hline
\end{tabular}

Individual $p$-values for the exact OLS linear model testing the VC hypothesis, and the additive $\left(P^{+}\right)$and multiplicative $\left(P^{\times}\right)$combined $p$-values $(n=6)$. Dependent variables were $\alpha$ amplitude, $\beta$ amplitude, $\beta / \alpha$ amplitude ratio, $\Phi^{1}$, and percent time spent in reinforcement state (\%tRS). Tests compared randomized trials A versus B. See text for details. In all tests EMG and EOG entered the model as covariates. Significant results (both $P^{+}$and $P^{\times} \leq 0.05$ ) are flagged with an asterisk. For individual $p$-values, italic font flags results in the predicted direction $(p<0.5)$.

TABLE II

RESULTS OF THE EL HYPOTHESIS

\begin{tabular}{r|c|c|c|c|c|c|c|c}
\hline EL; $\mathrm{n}=6$ & $\mathrm{~S} 1$ & $\mathrm{~S} 2$ & $\mathrm{~S} 3$ & $\mathrm{~S} 4$ & $\mathrm{~S} 5$ & $\mathrm{~S} 6$ & $\mathbf{P}^{+}$ & $\mathbf{P}^{\mathbf{x}}$ \\
\hline$\alpha$ & 0.3168 & 0.2681 & 0.3680 & 0.8333 & 0.0003 & 0.8041 & $\mathbf{0 . 2 8 6}$ & $\mathbf{0 . 0 2 1}$ \\
\hline$\beta^{*}$ & 0.3654 & 0.6000 & 0.2509 & 0.1103 & 0.0011 & 0.2747 & $\mathbf{0 . 0 2 3}$ & $\mathbf{0 . 0 0 9}$ \\
\hline$\beta / \alpha^{*}$ & 0.2757 & 0.2168 & 0.3681 & 0.7400 & 0.0001 & 0.6631 & $\mathbf{0 . 1 5 3}$ & $\mathbf{0 . 0 0 7}$ \\
\hline$\Phi^{1}$ & 0.3685 & 0.2100 & 0.3714 & 0.2200 & 0.0002 & 0.2419 & $\mathbf{0 . 0 1 1}$ & $\mathbf{0 . 0 0 3}$ \\
\hline$\% \mathrm{tRS}$ & 0.1418 & 0.6718 & 0.2521 & 0.2900 & 0.4785 & 0.6431 & $\mathbf{0 . 2 3 5}$ & $\mathbf{0 . 4 2 3}$ \\
\hline
\end{tabular}

Individual $p$-values for the exact OLS linear model testing the EL hypothesis, and the additive $\left(P^{+}\right)$and multiplicative $\left(P^{\times}\right)$combined $p$-values $(n=6)$. Dependent variables were $\alpha$ amplitude, $\beta$ amplitude, $\beta / \alpha$ amplitude ratio, $\Phi^{1}$, and percent time spent in reinforcement state (\%tRS). Tests evaluated linear trends across sessions. See text for details. In all tests EMG and EOG entered the model as covariates. Significant results (both $P^{+}$and $P^{\times} \leq 0.05$ ) are flagged with an asterisk. For individual $p$-values, italic font flags results in the predicted direction $(p<0.5)$.

the intention of the subjects, however, the additively combined $p$-value for the time spent in reinforcement state was significantly small $\left(P^{+}=0.042\right)$.

Results for the EL hypothesis displayed a similar pattern (see Table II). Again, regardless of the combination function considered, all combined $p$-values were in the expected direction. The $\beta / \alpha$ ratio was significant according to the multiplicative combining function $\left(P^{\times}=0.007\right)$, but not according to the additive one $\left(P^{\times}=0.153\right)$. However, the $\beta$ amplitude increased significantly over session $\left(P^{+}=0.023 ; P^{\times}=0.009\right)$, with one subject only (S2) displaying a clearly negative result. For the EC hypothesis the percent time spent in the reinforcement state did not change over session. Instead, the $\Phi^{1}$ function increased significantly $\left(P^{+}=0.011 ; P^{\times}=0.003\right)$, the trend being supported by the data of five out of six subjects. In general, it appears that subjects could achieve changes in raw power measurements more easily than changes in the feedback signal itself, or in the time spent in reinforcement state. These results may be explained by the fact that fractional changes need to continuously reverse sign as changes in one direction (e.g., increase in alpha power) cannot proceed indefinitely, and their long-term average must converge to zero. On the whole these results indicate that during adequate training, average current density power in the ACdc can increase or decrease according to the chosen protocol (EL) and that soon after the training, subject are able to temporarily shift the average power in the desired direction by an act of will (VC).

\section{DISCUSSIONAND CONCLUSION}

The decision to combine the $p$-values of the six subjects for both the volitional control and exercise learning hypotheses received substantial support in this LORETA neurofeedback ex- periment. While VC is largely ignored in the literature on neurofeedback, it is very important in our understanding of the neurofeedback learning process and is relevant to the development of BCIs. Indeed, communication with electronic devices, the goal of BCI systems, is intended as a volitional act. This finding suggests that operant conditioning theory is not necessary for explaining the human ability to self-regulate and/or voluntarily produce specific brain electrical activity. A shift in the $\beta / \alpha$ amplitude ratio over session was also supported by the experiment, although indirectly; changes in the ratio per se were not significant. However changes in alpha and beta were found in the desired direction, and the latter reached significance. EL is relevant for clinical application of neurofeedback, but it does not constitute a proof that the changes are permanent, that is, that they last once the session is ended. To test whether or not a change is persistent, one has to collect presession baselines and demonstrate learning curves with respect to the presession data. This latter kind of learning process, which we call long-term learning (LL), is the one that really matters in neurofeedback clinical applications.

Our findings suggest not only that a spatial filter (EEG inverse solution) can be applied to the feedback signal to train spatial specific brain regions, but also that the feedback signal can be based on a complex function of the raw power measurements. The former suggestion extends the applicability of neurofeedback techniques to deep neocortical regions. The latter implies that it is possible to overcome the subjective configuration of raw power measurements, yielding neurofeedback protocols identical for all individuals.

We assume that learning associated with neurofeedback is achievable for most but not all individuals. Actually, all we know is that experiments designed to demonstrate learning 
do not always succeed, and that the same phenomenon is observed in clinical practice. In this experiment, individual $p$-values (Table I and II) showed considerable variability. In future research, it would be important to understand under what circumstances the training is successful and under what circumstances it is not, and if the decisive factors for success are related to experimental variables and/or technical factors, to variables extraneous to the individual or to individual characteristics.

A recent advance in the LORETA method has been achieved introducing the standardized LORETA method (sLORETA). While LORETA has a low localization error, sLORETA has the theoretical ability to localize correctly single sources, as seen in noise-free point spread function simulations [60]. The implementation of sLORETA is recommended for future research.

While localization has been shown to be accurate in the absence of noise, for real EEG data, which is mixed with several source of intracranial and extracranial noise [61], localization accuracy is expected to decrease. Current research in real-time denoising algorithms [62], [63] will prove essential for the effective use of real-time brain research based on electromagnetic tomographic techniques.

Another limitation of the current method that needs to be addressed is the influence of the spatial smearing observed in LORETA and sLORETA current density reconstructions [25], [60]. Both the inverse methods achieve precision in source localization by sacrificing spatial resolution, i.e., the sources are reconstructed spatially dispersed even if they are point-like [24]-[27]. As a consequence, even if the ROI is spatially delimited, strong current densities in adjacent regions may influence the current density estimation within the ROI. If this influence is found to be important, weights could be applied to the appropriate rows of the inverse solution transformation matrix [25] in order to attenuate the influence of neighboring regions.

We demonstrated a possible implementation of neurofeedback based on the LORETA. We developed a method and we conducted some preliminary experimentation. In this paper, we addressed several methodological concerns that arise in real-time EEG applications and we proposed some solutions. The results we obtained are encouraging but by no means conclusive. In this research, we focused on methodological aspects. The advantage of tomographic neurofeedback over traditional (scalp) neurofeedback still needs to be assessed. In the future, we predict the use of stereoscopic feedback, high-impedance electrodes and other sophisticated apparatus to facilitate the delivery of feedback. An immersive virtual three-dimensional brain navigation system utilizing electromagnetic data feedback is currently under development. In addition to providing more immersive and realistic feedback experiences, the integration of several sensory modalities seems particularly promising. We hypothesize that the more complex the feedback sensory experience, the more likely the electrophysiological processes of interest will be engaged. Henceforth, speed and precision of learning may be improved considerably by means of variations on this proposed technology. Furthermore, we think that more informative feedback signals could be employed in future research. The increase or decrease of power, power asymmetry or coherence, the typical focus of current neurofeedback experiments and clinical applications, may provide too little information about the brain's electrical activity. Insufficient feedback information may actually impede the learning process. The $\Phi^{1}$ function we here introduced proved useful. Incorporating other features such as multivariate autoregressive analysis or joint time-frequency-space correlation [53] may also provide significant advantages.

\section{ACKNOWLEDGMENT}

The authors would like to thank J. Rothove for his assistance during experimentation and Dr. M. Koca for his comments about the draft of the paper.

\section{REFERENCES}

[1] D. R. Engstrom, P. London, and J. T. Hart, "Hypnotic susceptibility increased by EEG alpha training," Nature, vol. 227, pp. 1261-1262, 1970

[2] D. P. Nowlis and J. Kamiya, "The control of electroencephalographic alpha rhythms through auditory feedback and the associated mental activity," Psychophysiology, vol. 6, no. 4, pp. 476-484, 1970.

[3] T. A. Travis, C. Y. Kondo, and J. R. Knott, "Alpha conditioning: A controlled study," J. Nerv. Mental Dis., vol. 158, pp. 163-173, 1974.

[4] M. Barabasz and A. Barabasz, "Attention deficit disorder: Diagnosis, etiology and treatment," Child Study J., vol. 26, no. 1, pp. 1-37, 1996.

[5] M. Linden, T. Habib, and V. Radojevic, "A controlled study of the effects of EEG biofeedback on cognition and behavior of children with attention deficit disorder and learning disabilities," Biofeedback Self-Regulat., vol. 21, no. 1, pp. 35-49, 1996.

[6] J. F. Lubar, "Discourse on the development of EEG diagnostics and diofeedback for attention-deficit/hyperactivity disorders," Biofeedback Self-Regulat., vol. 16, no. 3, pp. 201-225, 1991.

[7] - "Neocortical dynamics: Implications for understanding the role of neurofeedback and related techniques for the enhancement of attention," Appl. Psychophysiol. Biofeedback, vol. 22, no. 2, pp. 111-126.

[8] J. F. Lubar and M. N. Shouse, "EEG and behavioral changes in a hyperkinetic child concurrent with training of the sensorimotor rhythms (SMR)," Biofeedback Self-Regulat., vol. 1, no. 3, pp. 293-306, 1976.

[9] T. Rossiter, "Neurofeedback for AD/HD: A ratio feedback case study and tutorial," J. Neurotherapy, vol. 6, no. 3, pp. 9-35, 2003.

[10] M. A. Tansey and R. L. Bruner, "EMG and EEG biofeedback training in the treatment of a 10-years-old hyperactive boy with a developmental reading disorder," Biofeedback Self-Regulat., vol. 8, no. 1, pp. 25-37, 1983.

[11] J. P. Rosenfeld, "An EEG biofeedback protocol for affective disorders," Clin. Electroencephalogr., vol. 3, no. 1, pp. 7-12, 2000.

[12] J. F. Lubar and W. W. Bahler, "Behavioral management of epileptic seizures following EEG biofeedback training of the sensorimotor rhythm," Biofeedback Self-Regulat., vol. 1, no. 1, pp. 77-104, 1976.

[13] J. F. Lubar, H. S. Shabsin, S. E. Netelson, G. S. Holder, S. F. Whitsett, W. E. Pamplin, and D. I. Krulikowski, "EEG operant conditioning in intractable epileptic," Arch. Neurol., vol. 38, pp. 700-704, 1981.

[14] M. B. Sterman, "Neurophysiologic and clinical studies of sensorimotor EEG biofeedback training: Some effects on epilepsy," Sem. Psych., vol. 5, no. 4 , pp. 507-525, 1973 .

[15] — "EEG biofeedback: physiological behavior modification," Neurosci. Biobehav. Rev, vol. 5, pp. 405-412, 1981.

[16] P. G. Swingle, "Neurofeedback treatment of pseudoseizure disorder," Biol. Psych., vol. 44, pp. 1196-1199, 1998.

[17] K. E. Thornton, "The improvement/rehabilitation of auditory memory functioning with EEG biofeedback," NeuroRehab., vol. 17, pp. 69-80, 2002.

[18] N. C. Moore, "A review of EEG biofeedback treatment of anxiety disorders," Clin. Electroencephalogr., vol. 31, no. 1, pp. 1-6, 2000.

[19] L. C. James and R. A. Folen, "EEG biofeedback as a treatment for chronic fatigue syndrome: A controlled case report," Behav. Med., vol. 22, pp. 77-81, 1996.

[20] A. Kübler, B. Kotchoubey, J. Kaiser, J. R. Wolpaw, and N. Birbaumer, "Brain-computer communication: unlocking the locked in," Psych Bull., vol. 127, no. 3, pp. 358-375, 2001. 
[21] B. Kotchoubey, A. Kubler, U. Strehl, H. Flor, and N. Birbaumer, "Can humans perceive their brain states?," Conscious. Cogn., vol. 11, no. 1, pp. 98-113, Mar. 2002.

[22] P. L. Nunez, Neocortical Dynamics and Human EEG Rhythms. Oxford, U.K.: Oxford Univ. Press, 1995.

[23] P. L. Nunez and R. B. Silberstein, "On the relationship of synaptic activity to macroscopic measurements: Does co-registration of EEG with fMRI make sense?," Brain Topogr., vol. 13, no. 2, pp. 79-96, 2000.

[24] R. D. Pascual-Marqui, C. M. Michel, and D. Lehmann, "Low resolution electromagnetic tomography: a new method for localizing electrical activity in the brain," Int. J. Psychophysiol., vol. 18, pp. 49-65, 1994.

[25] R. D. Pascual-Marqui, "Review of methods for solving the EEG inverse problem," Int. J. Bioelectromagn., vol. 1, no. 1, pp. 75-86, 1999a.

[26] _ - "Reply to comments made by Grave de Peralta Menendez and S. I. Gonzalez Andino," Int. J. Bioelectromagn., vol. 1, no. 2, 1999 b.

[27] — _ "Reply to comments by Hämäläinen, Ilmonieni, and Nunez," in Source Localization: Continuing Discussion on the Inverse Problem, W. Skrandies, Ed: ISBET Newsletter, 1995, vol. 6, pp. 16-28.

[28] R. D. Pascual-Marqui, M. Esslen, K. Kochi, and D. Lehmann, "Functional imaging with low resolution brain electromagnetic tomography (LORETA): A review," Meth. Findings Exp. Clin. Pharmacol., vol. 24C, pp. 91-95, 2002a.

[29] _ "Functional imaging with low resolution brain electromagnetic tomography (LORETA): Review, new comparisons, and new validation," Jpn. J. Clin. Neurophysiol., vol. 30, pp. 81-94, 2002 b.

[30] J. Bosch-Bayard, P. Valdés-Sosa, T. Virues-Alba, E. Aubert-Vázquez, E. R. John, T. Harmony, J. Riera-Diaz, and N. Trujillo-Barreto, "3-D statistical parametric mapping of EEG source spectra by means of variable resolution electromagnetic tomography (VARETA)," Clin. Electroencephalogr., vol. 32, pp. 47-61, 2001.

[31] T. Isotani, H. Tanaka, D. Lehmann, R. D. Pascual-Marqui, K. Kochi, N. Saito, T. Yagyu, T. Kinoshita, and K. Sasada, "Source localization of EEG activity during hypnotically induced anxiety and relaxation," Int. J. Psychophysiol., vol. 41, pp. 143-153, 2001.

[32] J. F. Lubar, M. Congedo, and J. H. Askew, "Low-resolution electromagnetic tomography (LORETA) of cerebral activity in chronic depressive disorder," Int. J. Psychophysiol., vol. 49, no. 3, pp. 175-185, Sept. 2003.

[33] D. Pizzigalli, R. D. Pascual-Marqui, J. B. Nitschke, T. R. Oakes, C. L. Larson, and H. C. Abercrombie et al., "Anterior cingulate activity as predictors of degree of treatment response in major depression: Evidence from brain electrical tomography analysis," Amer. J. Psych., vol. 158, pp. 405-415, 2001.

[34] A. Fernández-Bouzas, T. Harmony, J. Bosch-Bayard, E. AubertVázquez, T. Fernández, and T. P. Valdés-Sosa et al., "Source of abnormal EEG activity in the presence of brain lesions," Clin. Electroencephalogr., vol. 30, pp. 46-52, 1999.

[35] M. Fuchs, M. Wagner, T. Köhler, and H. Wischmann, "Linear and nonlinear current density reconstructions," J. Clin. Neurophysiol., vol. 16, no. 3, pp. 267-295, 1999.

[36] L. S. Prichep, E. R. John, and M. Tom, "Localization of deep white matter lymphoma using VARETA: A case study," Clin. Electroencephalogr., vol. 32, pp. 62-66, 2001.

[37] G. A. Worrell, T. D. Lagerlund, F. W. Sharbrough, B. H. Brinckmann, N. E. Bucacker, K. M. Cicora, and T. J. O'Brien, "Localization of the epileptic focus by low-resolution electromagnetic tomography in patients with a lesion demonstrated by MRI," Brain Topogr., vol. 12, pp. 273-282, 2000.

[38] J. Talairach and P. Tournoux, Co-Planar Stereotaxic Atlas of the Human Brain. Stuttgart, Germany: Thieme, 1988.

[39] J. L. Lancaster, L. H. Rainey, J. L. Summerlin, C. S. Freitas, P. T. Fox, and A. Evans et al., "Automated labeling of the human brain: A preliminary report on the development and evaluation of a forward-transform method," Human Brain Map., vol. 5, pp. 238-242, 1997.

[40] J. L. Lancaster, M. G. Woldorff, L. M. Parsons, M. Liotti, C. S. Freitas, and L. Rainey et al., "Automated talairach atlas labels for functional brain mapping," Human Brain Map., vol. 10, pp. 120-131, 2000.

[41] V. L. Towle, J. Bolaños, D. Suarez, K. Tan, R. Grzeszczuk, and D. N. Levin et al., "The spatial location of EEG electrodes: Locating the best fitting sphere relative to cortical anatomy," Electroencephalogr. Clin. Neurophysiol., vol. 86, pp. 1-6, 1993.

[42] S. S. Yoo and F. A. Jolesz, "Functional MRI for neurofeedback; feasibility study on a hand motor task," Neuroreport, vol. 13, no. 11, pp. 1377-1381, Aug 2002.

[43] C. J. Harland, T. D. Clark, and R. J. Prance, "Remote detection of human electroencephalograms using ultrahigh input impedance electric potential sensors," Appl. Phys. Lett., vol. 81, pp. 3284-3286, 2002.
[44] M. Congedo and J. F. Lubar, "Parametric and nonparametric analysis of qEEG: Normative database comparisons in electroencephalography, a simulation study on accuracy," J. Neurotherapy, vol. 7, no. 3, pp. 1-29, 2003.

[45] J. L. Blom and M. Anneveldt, "An electrode cap tested," Electroencephalogr. Clin. Neurophysiol., vol. 54, pp. 591-594, 1982.

[46] G. Bush, J. A. Frazier, S. L. Rauch, L. J. Seidman, P. J. Whalen, and M. A. Jenike et al., "Anterior cingulate cortex dysfunction in attention deficit/hyperactivity disorder revealed by fMRI and the counting stroop," Biol. Psych., vol. 45, no. 12, pp. 1542-1552, 1999.

[47] O. Devinsky, M. J. Morrell, and B. A. Vogt, "Contributions of anterior cingulate to behavior," Brain, vol. 118, pp. 279-306, 1995.

[48] R. J. Barry, A. R. Clarke, and S. J. Johnston, "A review of electrophysiology in attention-deficit/hyperactivity disorder: I. Qualitative and quantitative electroencephalography," Clin. Neurophysiol., vol. 114, pp. 171-183, 2003.

[49] P. L. Nunez, B. M. Wingeier, and R. B. Silberstein, "Spatial-temporal structures of human alpha rhythms: Theory, microcurrent sources, multiscale measurements, and global binding of local networks," Human Brain Map., vol. 13, no. 3, pp. 125-164, 2001.

[50] A. F. Leuchter, S. H. J. Uijtdehaage, I. A. Cook, R. O'Hara, and M. Mandelkern, "Relationship between brain electrical activity and cortical perfusion in normal subjects," Psych. Res.: Neuroimag. Sect., vol. 90, pp. $125-140,1999$.

[51] J. L. Hernández, P. Valdés, R. Biscay, T. Virues, S. Szava, and J. Bosch et al., "A global scale factor in brain," Int. J. Neurosci., vol. 76, pp. 267-278, 1994.

[52] J. V. Hardt and J. Kamiya, "Conflicting results in EEG alpha feedback studies. Why amplitude integration should replace percent time," Biofeedback Self-Regulat., vol. 1, no. 1, pp. 63-75, 1976.

[53] T. Ebrahimi, J. M. Vesin, and G. Garcia, "Brain-computer interface. A new frontier in multimedia communication," Signal Processing Mag., to be published.

[54] E. S. Edgington, Randomization Tests, 3rd ed. New York: Marcel Dekker, 1995.

[55] F. Pesarin, Multivariate Permutation Tests. New York: Wiley, 2001.

[56] B. F. J. Manly, Randomization, Bootstrap and Monte Carlo Methods in Biology, 2nd ed. London, U.K.: Chapman \& Hall, 1997.

[57] B. S. Cade and J. D. Richards, User Manual for Blossom Statistical Software. Fort Collins, CO: U.S. Geological Survey, 1999.

[58] P. E. Kennedy and B. S. Cade, "Randomization tests for multiple regression," Commun. Statist. Simula., vol. 25, pp. 923-936, 1996.

[59] M. J. Anderson and P. Legendre, "An empirical comparison of permutation methods for tests of partial regression coefficients in a linear model," J. Statist. Comput. Simula., vol. 62, pp. 271-303, 1999.

[60] R. D. Pascual-Marqui, "Standardized low resolution brain electromagnetic tomography (sLORETA): Technical details," Meth. Findings Exp. Clin. Pharmacol., vol. 24D, pp. 5-12, 2002.

[61] S. A. Vorobyov and A. Cichocki, "Blind noise reduction for multisensory signals using ICA and subspace filtering, with application to EEG analysis," Biol. Cybern., vol. 86, pp. 293-303, 2002.

[62] A. Cichocki and S. Amari, Adaptive Blind Signal and Image Processing. New York: Wiley, 2003.

[63] A. Hyvärinen, J. Karhunen, and E. Oja, Independent Component Analysis. New York: Wiley, 2001.

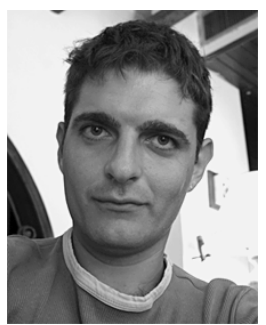

Marco Congedo was born in Italy, in 1972. He received the B.A. degree in psychology from the University of Padua, Padua, Italy, in 1998, and the M.A. and Ph.D. degrees in biological psychology (and a minor $\mathrm{Ph} . \mathrm{D}$. degree in statistics) from the University of Tennessee, Knoxville, in 2001 and 2003, respectively.

Currently, he is a Postdoctoral Fellow with the National Institute for Research in Informatics and Random Systems, Rennes, France. His research interests include basic EEG/MEG research, real-time neuroimaging, and multivariate statistics.

While he was a graduate student, Dr. Congedo twice received the Department of Psychology Award for Excellence in Scholarship and Graduate Research. $\mathrm{He}$ is a member of the International Society of Neuronal Regulation and of the Society of Applied Neuroscience. 


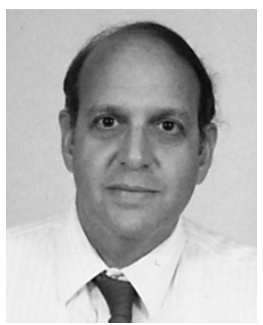

Joel F. Lubar received the B.S. and Ph.D. degrees from the Division of the Biological Sciences, Department of Biopsychology, University of Chicago, Chicago, IL.

He has been an Assistant Professor with the University of Rochester, Rochester, NY; a Senior Faculty Science Fellow with the National Science Foundation, UCLA School of Medicine, Los Angeles; a Visiting Professor at the Institute of Physiology, School of Medicine, University of Bergen, Bergen, Norway; and is currently a Full Professor with the University of Tennessee, Knoxville. He has published more than 100 papers, wrote many book chapters, and eight books in the area of neuroscience and applied psychophysiology. He has been a Regional Editor for the Journal Physiology and Behavior, and an Associate Editor for Biofeedback and Self Regulation, Associate Editor for the Journal of Neurotherapy.

Dr. Lubar was the President of the Academy of Certified Neurotherapists, which he incorporated into the Biofeedback Certification Institute of America EEG Specialty area. He has also been President of the EEG Division of the Association for Applied Psychophysiology and Biofeedback (AAPB), President of AAPB from 1996 to 1997, a member of the Editorial Board for the Journal of Applied Psychophysiology and Biofeedback. He is currently President of the International Society for Neuronal Regulation.

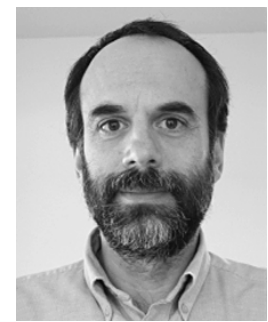

David Joffe was born in New York, in 1952. He received the B.A. degree in mathematics from the University of Colorado, Boulder, in 1980.

He has been involved in the founding of numerous biomedical companies and the development of many biomedical instruments. He is currently the Vice President of Research and Development at Lexicor Medical Technology, Boulder. His research interests include the development of real-time multichannel EEG signal processing algorithms.

Mr. Joffe received a Distinguished Achievement Award from the Neurofeedback Division of the Association for Applied Psychophysiology and Biofeedback in 2004. 